\title{
Secretory lgA's complex roles in immunity and mucosal homeostasis in the gut
}

\author{
NJ Mantis ${ }^{1,2}, \mathrm{~N} \mathrm{Rol}^{3}$ and B Corthésy ${ }^{3}$
}

\begin{abstract}
Secretory $\lg A(S \lg A)$ serves as the first line of defense in protecting the intestinal epithelium from enteric toxins and pathogenic microorganisms. Through a process known as immune exclusion, SlgA promotes the clearance of antigens and pathogenic microorganisms from the intestinal lumen by blocking their access to epithelial receptors, entrapping them in mucus, and facilitating their removal by peristaltic and mucociliary activities. In addition, SIgA functions in mucosal immunity and intestinal homeostasis through mechanisms that have only recently been revealed. In just the past several years, SIgA has been identified as having the capacity to directly quench bacterial virulence factors, influence composition of the intestinal microbiota by Fab-dependent and Fab-independent mechanisms, promote retrotransport of antigens across the intestinal epithelium to dendritic cell subsets in gut-associated lymphoid tissue, and, finally, to downregulate proinflammatory responses normally associated with the uptake of highly pathogenic bacteria and potentially allergenic antigens. This review summarizes the intrinsic biological activities now associated with SlgA and their relationships with immunity and intestinal homeostasis.
\end{abstract}

\section{INTRODUCTION}

As the most abundant class of antibodies found in the intestinal lumen of humans and in most other mammals, secretory $\operatorname{IgA}(\mathrm{SIg}$ A) has long been recognized as a first line of defense in protecting the intestinal epithelium from enteric pathogens and toxins. SIgA production against specific mucosal antigens is dependent on the sampling by Peyer's patch $M$ cells, processing by antigen-presenting cells such as dendritic cells (DCs), T-cell activation, and ultimately B-cell class switch recombination in gut-associated lymphoid tissues (GALTs), mesenteric lymph nodes, and possibly neighboring lamina propria. ${ }^{1,2}$ Isolated lymphoid follicles in the small and large intestines also function in the induction of mucosal immune responses. ${ }^{3}$ Multiple cytokines, including interleukin (IL)-4, transforming growth factor- $\beta$, IL-5, IL-6, and IL-10 are instrumental in intestinal stimulation of SIgA production. A subset of these cytokines, notably transforming growth factor- $\beta$ and IL-10, is also required for maintaining mucosally induced tolerance, thus establishing one of the many links between SIgA production, immunity, and intestinal homeostasis.

This review highlights our current understanding of SIgA's many (recently revealed) functions in mucosal immunity and intestinal homeostasis. As SIgA essentially resides within an external environment (i.e., the intestinal lumen), it must combat microbial infections through mechanisms that are fundamentally different from those used by antibodies in systemic compartments. Whereas IgG promotes killing and clearance of pathogenic bacteria through coordinated activity of complement and Fc-mediated uptake by macrophages and neutrophils, it is generally assumed that SIgA acts primarily through receptor blockade, steric hindrance, and/or immune exclusion. We summarize evidence for each of these activities as revealed through the use of animal models, but argue that the mechanisms underlying SIgA-mediated immunity are in fact much more complex than previously appreciated.

With respect to intestinal homeostasis, we make the case that SIgA's multifaceted roles in controlling inflammation and in regulating immune responses to certain dietary antigens, commensal microflora, and enteric pathogens are only beginning to be understood. The past several years have seen an emergence of evidence that indicates that SIgA influences the composition of the intestinal microbiota, promotes the uptake and delivery of antigens from the intestinal lumen to DC subsets located in GALTs, and influences inflammatory responses normally associated with the uptake of highly pathogenic bacteria and potentially allergenic antigens. Owing to space limitations, this

${ }^{1}$ Division of Infectious Diseases, Wadsworth Center, New York State Department of Health, Albany, New York, USA. ²Biomedical Sciences Program, University at Albany School of Public Health, Albany, New York, USA. ${ }^{3}$ R\&D Laboratory of the Division of Immunology and Allergy, Centre Hospitalier Universitaire Vaudois, Lausanne, Switzerland. Correspondence: NJ Mantis or B Corthésy (nmantis@wadsworth.org or blaise.corthesy@chuv.ch) 
review will be mainly focused on SIgA's activities within the intestinal lumen; we will not discuss the capacity of polymeric IgA to neutralize pathogens intracellularly during transepithelial transport or to promote excretion of antigens present in the lamina propria.

\section{MULTIPLE NEUTRALIZING PROPERTIES OF SIgA AT GUT MUCOSAL SURFACES \\ Blocking attachment to epithelial cells by steric hindrance and binding to receptor-recognition domains}

SIgA is capable of interfering with the earliest steps in the infection process by virtue of its ability to block toxins and pathogens from adhering to the intestinal epithelium. ${ }^{4-9}$ One of the best examples of this mode of protection involves cholera toxin (CT), the toxin responsible for severe secretory diarrhea associated with Vibrio cholerae infection. In mouse models, it has been demonstrated that SIgA is essential in protecting the intestinal epithelium from the effects of luminal CT exposure. ${ }^{9,10}$ Not surprisingly, mouse IgA monoclonal antibodies (mAbs) against the toxin's B subunit (CTB), a homopentameric molecule that binds to ganglioside $\mathrm{GM}_{1}$ on the apical surfaces of enterocytes, were sufficient to prevent $\mathrm{CT}$ attachment to polarized intestinal epithelial cell monolayers in vitro. These same IgA mAbs protected neonatal mice from CT-induced secretory diarrhea, weight loss, and death. ${ }^{11}$ However, the mAbs did not directly interact with the $\mathrm{GM}_{1}$-binding site on CTB. Therefore, it was suggested that they likely interfere with CT binding to epithelial cells through a mechanism involving steric hindrance.

SIgA has also been shown to be capable of blocking pathogens from attaching to intestinal epithelial cells by direct recognition of receptor-binding domains, as demonstrated in the case of reovirus type 1 Lang. SIgA is required for full protection against intestinal reovirus type 1 Lang infection, as revealed through the use of IgA knockout mice. ${ }^{12}$ To investigate the molecular mechanism underlying SIgA-mediated immunity to reovirus, a panel of reovirus-specific IgA mAbs was screened for those that protected mice against an oral type 1 Lang challenge. Protection was conferred by IgA mAbs directed against the $\sigma 1$ protein, an adhesin fiber known to promote viral attachment to a number of epithelial cell types. ${ }^{13}$ The exact epitope recognized by one particular IgA mAb was localized to a $\sim 30$ amino-acid region of the $\sigma 1$ receptor-binding head domain, providing strong evidence that the $\mathrm{mAb}$ directly interferes with epithelial recognition. ${ }^{5}$ IgA mAbs against the other viral surface antigens, including the capsid protein, were not protective in the type 1 Lang peroral challenge model, underscoring the importance of epitope specificity in SIgA-mediated immunity. ${ }^{6}$

\section{Immune exclusion: agglutination, entrapment, and clearance} "Immune exclusion" generally refers to the ability of SIgA to prevent microbial pathogens and antigens such as toxins from gaining access to the intestinal epithelium through a stepwise series of events involving agglutination, entrapment in mucus, and/or clearance through peristalsis. ${ }^{14-16}$ Although immune exclusion has been recognized as a function of SIgA for nearly four decades, ${ }^{17}$ and often attributed as being an important component of protective immunity, ${ }^{18}$ very little is known about the specific details of the process.

Agglutination is the formation of macroscopic clumps of bacteria (or viruses) as the result of antibody-mediated crosslinking through polyvalent surface antigens. There are no reports in the literature to suggest that agglutination per se has any detrimental effect on microbial physiology or virulence. On the contrary, we (along with others) have reported that bacterial growth rates in culture are unaffected by agglutination. ${ }^{19,20}$ However, scanning and transmission electron microscopy analysis of Salmonella enterica serovar Typhimurium cross-linked by a protective IgA mAb ("Sal4") against the $\mathrm{O}$ antigen has revealed evidence of antibody-mediated distortion of the bacterial outer membranes (Figure 1), secretion of a capsular exopolysaccharide, and alterations in bacterial gene expression (S. Forbes, J. Amarasinghe, J. Dornenburg, and $\mathrm{N}$. Mantis, manuscript in preparation). Cross-linking of Salmonella typhimurium with antibodies against the flagella did not elicit any ultrastructural changes in membrane integrity, demonstrating that agglutination is qualitatively different depending on the epitope recognized by the agglutinating antibody, and that some antibodies may have immediate effects on bacterial physiology and gene expression.

Work by Phalipon, Corthésy, and colleagues ${ }^{21,22}$ has examined in mouse and rabbit model systems the capacity of SIgA to entrap bacterial pathogens in the mucus layer overlying respiratory and intestinal epithelia in vivo. Through the use of light microscopy, immunohistochemistry, and autoradiography, it was shown that a murine IgA mAb (IgAC5) specific for the $\mathrm{O}$ antigen of Shigella flexneri readily entrapped the bacterium within a thin layer of mucus overlying the epithelium. This activity was considerably greater when IgAC5 was complexed with bound secretory component (SC), apparently because the oligosaccharide side chains of SC associate with mucus. The mucus layer in the mouse and human small and large intestines is complex, ${ }^{23}$ and defining the specific molecular interactions between SIgA and individual components of the mucus layer will be necessary to fully understand the mechanisms that govern immune exclusion. Moreover, it is unclear to what degree (if any) SIgA promotes immune exclusion of commensal bacteria.

Although the capacity of specific SIgAs to entrap bacteria in the intestinal mucus in experimental settings is undeniable, it remains to be determined to what degree immune exclusion contributes to protective immunity to other enteropathogens, especially viruses. Indeed, it has been argued that any SIgA capable of binding to the surface of a pathogen is theoretically sufficient to intercept that pathogen in the intestinal lumen and to reduce or even block its attachment to the intestinal epithelium. ${ }^{24}$ However, coating of rotavirus or reovirus with "nonneutralizing" IgA mAbs in the intestinal lumen of mice is not sufficient to block infection. ${ }^{6,25}$ Rather, the primary determinant of protective immunity correlated with epitope specificity, thus challenging the importance of immune exclusion in mucosal immunity to viruses. 

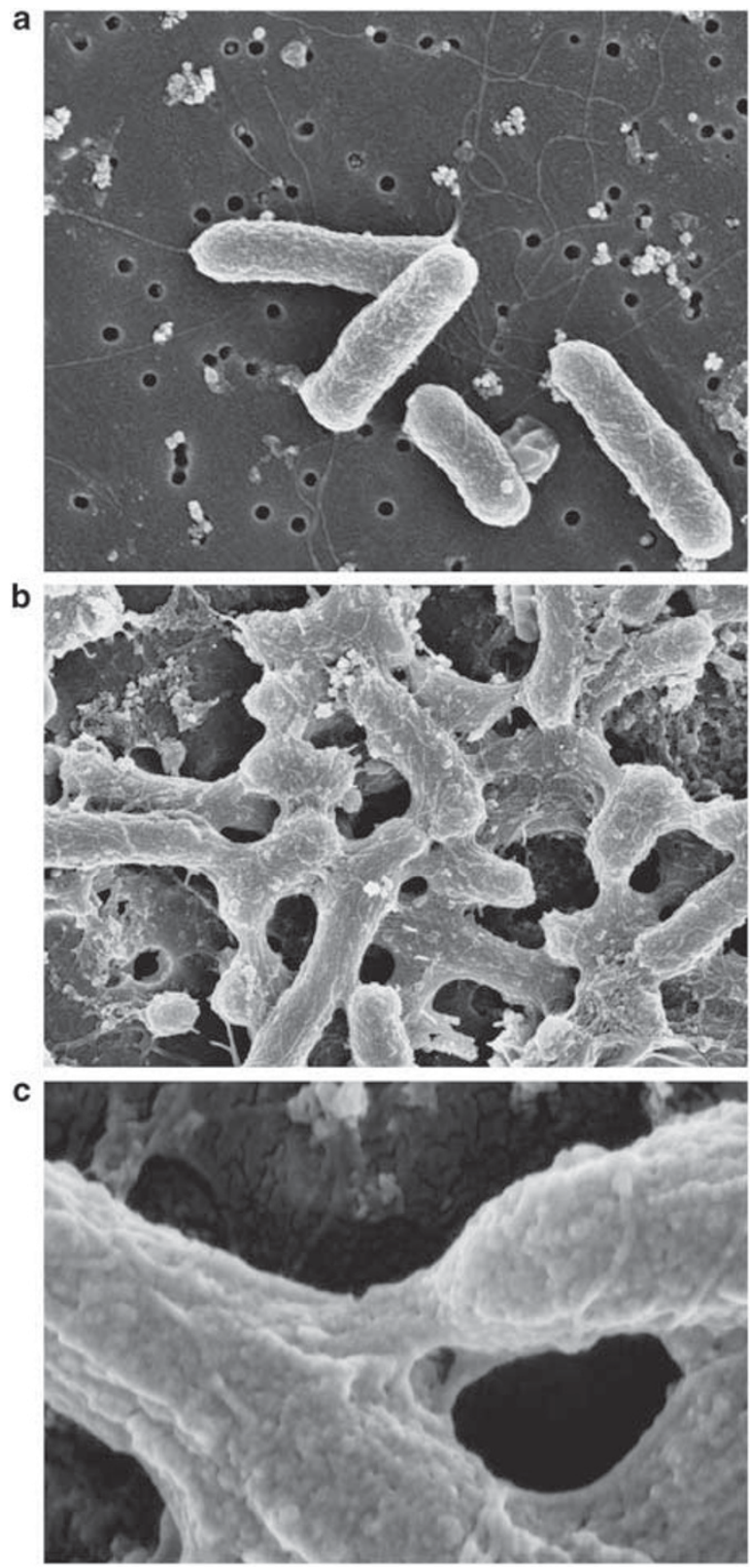

Figure 1 IgA-mediated agglutination of S. typhimurium is accompanied by gross changes in cell shape. Mid-log phase cultures of $S$. typhimurium strain $14028 \mathrm{~S}$ were exposed to Sal $4 \mathrm{mAb}\left(5 \mu \mathrm{g} \mathrm{ml}^{-1}\right)$ for $45 \mathrm{~min}$ and then subjected to scanning electron microscopy. (a) S. typhimurium control cells not treated with Sal4; (b and c) cells treated with Sal4, at (panel b) low and (panel c) high magnification. The gross changes in cell shape and the bridging that occurs between cells in the presence of Sal4 (panels $\mathbf{b}$ and $\mathbf{c}$ ) but not in the absence of Sal4 (panel a) must be noted. Figure kindly provided by $\mathrm{Dr}$ Steve Forbes. mAb, monoclonal antibody.

\section{Direct effects of SIgA on bacterial virulence}

It has been recognized for years that neither immune exclusion nor direct interference with attachment to epithelial receptors can fully account for the protective effects observed by a number of IgA mAbs, such as those against the $\mathrm{O}$ antigens of $V$. cholerae, S. typhimurium, and S. flexneri. ${ }^{11,19,22,26,27}$ This prompted us to examine the possibility that IgA may have a direct effect on bacterial virulence. We found that the binding of a murine $\operatorname{IgA}$
$\mathrm{mAb}$ (IgAC5) to the $\mathrm{O}$ antigen of S. flexneri suppressed activity of the bacterial type 3 secretion system that is necessary for S. flexneri to gain entry into intestinal epithelial cells. ${ }^{28}$ The suppressive effect of IgAC5 on type 3 secretion activity was rapid (5-15 $\mathrm{min}$ ) and coincided with a partial reduction in the bacterial membrane potential and intracellular ATP levels. Although IgAC5 is neither bacteriostatic nor bactericidal, it clearly has the capacity to selectively "quench" certain virulence factors. It remains to be determined whether other IgA antibodies share this trait.

\section{Fab-independent interaction between natural SIgA and intestinal pathogenic and commensal bacteria}

SIgA is also capable of preventing pathogen and toxin attachment to epithelial surfaces, independent of the antibody variable region. In this respect, SIgA can be considered a component of the innate immune system. Both the IgA heavy chain $(\mathrm{Fc} \alpha)$ and SC are heavily glycosylated. As the oligosaccharide side chains present on SIgA share a high degree of similarity with those on the luminal face of the intestinal epithelium, it has been proposed that $\operatorname{IgA}$ and $\mathrm{SC}$ (free or bound to $\operatorname{Ig} \mathrm{A}$ ) can effectively serve as competitive inhibitors ("decoys") of pathogen binding to host cells. ${ }^{29-35}$ For example, SIgA in concentrations at or below those found in human milk inhibited the binding of Clostridium difficile toxin A to purified enterocyte brush border membrane receptors. ${ }^{29}$ Toxin A bound to free SC as strongly as it did to the heavy and light chains of IgA. Perrier et al. ${ }^{32}$ identified the galactose and sialic acid residues on free $\mathrm{SC}$ as being primarily responsible for blocking toxin A attachment to epithelial cell monolayers. SC's activities are in fact quite broad, as free SC has been shown to serve as a decoy receptor for other pathogens, including enteropathogenic Escherichia coli by binding to intimin ${ }^{32}$ and Streptococcus pneumoniae through interaction with the choline-binding protein A. ${ }^{36}$ Along the same lines, a recent study has shown that mannose residues present on SIgA (but not serum IgG or IgM) are implicated in the inhibition of V. cholerae biofilm formation. ${ }^{37}$

The interaction between SIgA and commensal bacteria involves Fab- and Fc-independent structural motifs, featuring bound SC as a crucial partner. Removal of glycans present on free SC or bound in SIgA resulted in a drastic decrease in the interaction with Gram-positive bacteria, indicating the essential role of carbohydrates in the process. ${ }^{38}$ Coating of commensal microorganisms by SIgA may favor gut colonization and education of the newborn's mucosal immune system toward antigens associated with symbiotic partners. For example, using human intestinal epithelial Caco- 2 cell grown as polarized monolayers, we found that association of a Lactobacillus or a Bifidobacterium with non-specific SIgA enhanced probiotic adhesion by a factor of $\geqslant 3.4$-fold. Moreover, SIgA affected epithelial permeability, signaling events involved in nuclear factor- $\kappa \mathrm{B}$ nuclear translocation, production of $\mathrm{pIgR}$, and induction of immune mediators. ${ }^{39}$ Taken together, these observations suggest that although sugarmediated non-specific recognition occurs, its highly plastic, combinatorial nature still permits a selective interaction with commensal, non-pathogenic, and pathogenic bacteria. 


\section{ROLE OF SIgA IN HOMEOSTASIS OF THE INTESTINAL MICROBIOTA \\ Induction of SIgA by neonatal exposure to commensal microorganisms}

Immediately after birth, mammals are exposed to microbes associated with the external and maternal environments. The transition from a sterile environment to a highly colonized environment is accompanied by concomitant exposure of the newborn's gastrointestinal tract to maternal IgA antibodies acquired through breast feeding. Natural and specific SIgA antibodies in breast milk are capable of binding commensal bacteria and may be involved in the progressive, controlled establishment of the newborn's microbiota. ${ }^{40,41}$ The microbiota, in turn, stimulates maturation of GALTs, resulting in the production of IgA with both a limited affinity and repertoire to redundant epitopes on gut microorganisms. ${ }^{42-44}$ By direct visualization of a fluorescently labeled commensal bacterium administered in the form of an SIgA-based complex into the intestines of mice, we observed both preserved association with the antibody and specific targeting to, and passage across, Peyer's patch $\mathrm{M}$ cells (Figure 2). This observation suggests that SIgA, by virtue of its ability to associate with commensal bacteria and promote their uptake through $\mathrm{M}$ cells (see below), may have an important role in controlling the sampling of commensal bacteria in the form of SIgAimmune complexes by GALTs (N. Rol, L. Favre, J. Benyacoub, and B. Corthésy, submitted for publication).

The past several years have yielded important information about the mechanisms involved in intestinal IgA response against commensal microorganisms. It has been proposed that in mice, a proportion of the specific SIgA against commensal bacteria are induced in a T cell-independent pathway, independent of the development of follicular lymphoid structures. ${ }^{45,46}$ Subsequent studies in mice revealed that commensal bacteria persist in Peyer's patch DCs, contributing to induction of local specific immune responses that limit dissemination no farther than the mesenteric lymph nodes, ultimately preventing systemic spread. ${ }^{47}$ Recently, Hapfelmeier et al. ${ }^{48}$ reported using a "reversible" germ-free mouse model that the intestinal-specific IgA response against commensal bacteria (i) requires a high threshold for induction $\left(\sim 10^{9}\right.$ bacteria), (ii) has a slow onset ( $\geqslant 14$ days) with a long half-life ( $>16$ weeks), and (iii) constantly adapts to the predominant commensal species in the intestinal lumen. However, it remains an open question as to whether this type of selective SIgA response actually reflects the situation in conventionally raised mice, which encounter a complex and diverse microbiota in the context of a fully matured GALTs.

\section{Involvement of SIgA in the control of commensal microorganisms}

Different model systems have been used to investigate the role of SIgA on intestinal homeostasis. Among them are mouse strains deficient in AID (activation-induced cytidine deaminase), which are unable to class switch from IgM to IgA. AID knockout mice were shown to have profound increases in the number of nonpathogenic, anaerobic bacteria throughout their small intestines, as well as hyperplasia of isolated lymphoid follicles. ${ }^{49}$ As $\mathrm{AID}^{-/-}$

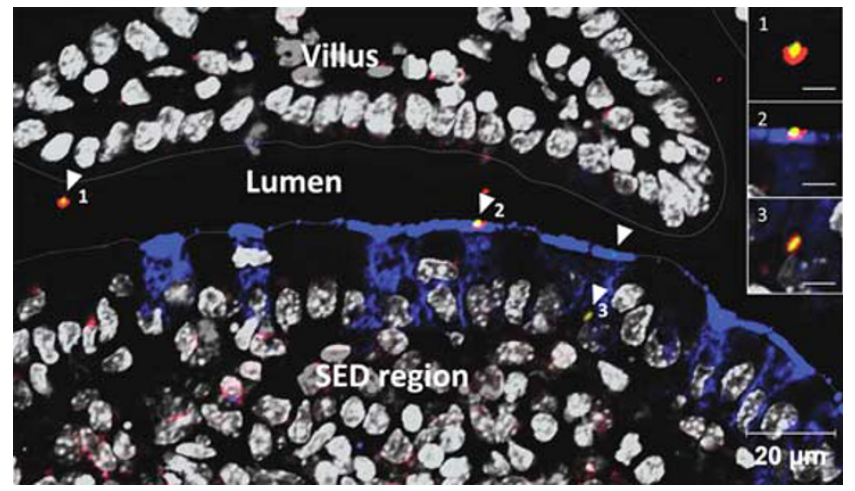

Figure 2 Entry of commensal bacteria coated with SIgA in a Peyer's patch through $\mathrm{M}$ cells. Image acquired by laser scanning confocal microscopy. Lactobacillus rhamnosus labeled with FITC was injected in the form of SIgA-based complexes into a mouse-ligated ileal loop comprising a single Peyer's patch. After a 2-h incubation, the tissue was removed and cryosectioned. M cells (blue), SlgA (red), and cell nuclei (gray) were stained with UEA-1, anti-SC antibodies, and DAPI, respectively. Arrowheads indicate bacteria in the form of SIgA-based complexes. The appearance of SlgA-based complexes in the lumen, at the surface of $\mathrm{M}$ cells, and transiting through an $\mathrm{M}$ cell reflects the various steps in the passage from the lumen to the SED region. Bars in insets represent $5 \mu \mathrm{m}$. DAPI, 4'-6-diamidino-2-phenylindole; FITC, fluorescein isothiocyanate; SED, subepithelial dome; SIgA, secretory IgA; UEA-1, Ulex europaeus agglutinin-1.

mice do not have functional SIgM, this study demonstrated that SIgA is essential in preventing hyper-stimulation of the mucosal and systemic immune systems. However, it has been noted that SIgM has compensatory activities in selectively IgA-deficient mice, possibly explaining why these mice are "healthy" under normal laboratory conditions. These observations in mice can be compared with human IgA deficiency in which a maturation defect in B cells is commonly observed. ${ }^{50}$ IgA-deficient patients are generally asymptomatic, but do exhibit a tendency to develop gastrointestinal disorders such as celiac disease $(\mathrm{CD})^{51}$ and allergies. ${ }^{52}$ IgA-deficient patients often have airway infections; however, these problems are mainly seen because compensatory SIgM is lacking in the airways (in contrast to the gut). ${ }^{53}$ Taken together, this supports the notion that adaptive SIgA responses may allow the host to respond to fluctuations in commensal bacteria without eliciting a deleterious response, and thus favor mucosal homeostasis. ${ }^{54}$

The dynamics between the commensal microbiota and SIgA are likely highly complex, considering that a considerable proportion (24-74\%) of the microbiota is coated with SIgA. ${ }^{55,56}$ Peterson et al. ${ }^{57}$ used an ingenious germ-free mouse model to directly examine the impact of SIgA on host-commensal interactions. The authors first produced an IgA mAb against the capsular polysaccharide of Bacteroides thetaiotaomicron. Germ-free, immunodeficient mice secreting this particular IgA mAb into the intestinal lumen by virtue of a hybridoma "backpack" were then challenged with $B$. thetaiotaomicron and both the host's and the commensal's responses were analyzed. The authors found that $B$. thetaiotaomicron, in the absence of specific anticapsular SIgA, elicited a robust oxidative stress response in the host. The presence of SIgA antibodies suppressed this response, 
thereby underscoring SIgA's potential to dampen deleterious host responses to commensal microbiota.

An interesting observation from the study by Peterson et al. ${ }^{57}$ was that the capsular polysaccharide-specific IgA $\mathrm{mAb}$ reduced, but did not prevent $B$. thetaiotaomicron from colonization of the mouse gut. Thus, under steady-state conditions, the presence of specific SIgA antibodies against surface antigens does not necessarily lead to bacterial clearance. Zitomersky et al. ${ }^{58}$ performed a longitudinal study of Bacteroidales species in 15 healthy adults over a period of a year and reached a similar conclusion. In fact, it has been argued that bacteria that bind SIgA may actually have a selective advantage in the gut. ${ }^{59}$ Bollinger et al. ${ }^{60,61}$ have shown that SIgA and mucin facilitate the formation of biofilms by nonpathogenic E. coli on epithelial cell monolayers grown in vitro. Biofilms have been proposed as a means by which endogenous microbiota colonize mucosal surface and ensure a steady-state growth rate in the intestinal lumen. ${ }^{62}$ The association of SIgA with biofilm formation in the gut has been demonstrated using sections from rat, baboon, and human tissues. ${ }^{63}$ Fluorescence in situ hybridization analysis has revealed that Lactobacilli species establish biofilms in different parts of the gastrointestinal tract, although it remains to be determined whether SIgA antibodies are involved in the process. ${ }^{64}$

\section{RETRO-TRANSPORT OF SIgA ACROSS THE INTESTINAL EPITHELIUM}

SIgA-based transport of immune complexes by M cells

It has been known for some time that Peyer's patch $\mathrm{M}$ cells selectively bind SIgA and SIgA-immune complexes. ${ }^{65,66}$ Although there is evidence for a SIgA-specific receptor on M cells, this receptor has not been identified. Therefore, the exact mechanism by which $M$ cells selectively internalize SIgA-antigen complexes in the face of a large excess of free SIgA present in the intestinal lumen remains unknown. Nonetheless, we recently provided evidence that SIgA undergoes conformational changes following antigen binding. ${ }^{67}$ Using specific SIgA antibodies with antigens of various sizes and complexity, we found that SIgA protease sensitivity profiles were altered upon antigen engagement, presumably reflecting differences in heavy chain backbone conformations. The conformational changes induced upon antigen interaction resulted in enhanced binding of SIgA to cellular receptors (FcaRI and pIgR), as compared with free (unbound) SIgA. These data reveal that antigen recognition by SIgA triggers structural changes in the immunoglobulin that result in enhanced receptor-binding properties. It remains to be determined whether this is relevant to $\mathrm{M}$ cell recognition of SIgA-based immune complexes.

\section{Consequences of SIgA-based immune complex uptake by M cells}

The recognition that SIgA-based immune complexes are transported by Peyer's patch $\mathrm{M}$ cells into the subepithelial dome (SED) regions has led us to investigate the role of "retro-translocation" in the regulation of intestinal immune responses. ${ }^{68}$ Oral delivery to mice of recombinant SIgA consisting of mouse polymeric IgA and human bound SC as a surrogate non-self antigen, triggered human bound SC-specific mucosal and systemic immune responses, as evidenced by antigen-specific serum and salivary antibody titers, T-cell proliferation in draining mesenteric lymph nodes and spleen, and pronounced expression of IL- 10 and transforming growth factor- $\beta$ by cells recovered from mesenteric lymph nodes. ${ }^{68}$ Furthermore, analysis of IgG isotypes and cytokine profiles demonstrated the tendency of recombinant SIgA immunization to induce a mixed Th1/Th2, tolerance-biased pattern of mucosal immune responses. It was also observed that recombinant SIgA induced migration of DCs from the SED region to the interfollicular region, a phenomenon indicative of DC activation. Although these responses were significantly less marked when compared with responses triggered upon mixing of human bound SC with the prototype mucosal adjuvant CT, they nonetheless reveal that IgA can function as an immunopotentiator in the mucosal environment.

\section{Reduction of pathogen-mediated proinflammatory responses by mucosal SIgA}

Besides being a weak immunopotentiator, there is evidence that SIgA may actively quench the capacity of certain antigens to elicit severe proinflammatory responses after uptake through Peyer's patch M cells. SIgA-coated S. flexneri, when injected into ligated ileal loops are detected in the SED region in close association with myeloid $\mathrm{CD} 11 \mathrm{c}^{+} \mathrm{CD} 11 \mathrm{~b}^{+} \mathrm{DCs}$, which are known to be tolerogenic. In the rabbit model (unlike the mouse), S. flexneri elicits a local severe acute inflammatory response that is reminiscent of that observed in humans. ${ }^{69}$ This model was used to assess the non-inflammatory capacity of protective SIgA at mucosal surfaces. ${ }^{21}$ Analysis of cytokine expression in Peyer's patches demonstrated that SIgA-coated S. flexneri results in downregulation of proinflammatory cytokines tumor necrosis factor- $\alpha$, IL- 6 , interferon- $\gamma$, while maintaining a sustained level of regulatory IL-10. This resulted in preservation of the integrity of the intestinal barrier, and suggests that under homeostatic conditions, SIgA exerts its anti-inflammatory effects by reducing bacteria-induced proinflammatory circuits (rather than promoting the onset of anti-inflammatory pathways). It is therefore tempting to speculate that the retro-transport of antigen-SIgA complexes is important for the maintenance of tolerance toward innocuous proteins, including allergens.

\section{SIgA as a scavenger of allergenic antigens}

Numerous animal and human studies have postulated that the secretory immune system is important in controlling allergic symptoms. Inhibition of allergens in the airways occurred with the abundant SIgA found in mucosal secretions and contributed toward limiting the access of allergen to the lamina propria and thus the inflammatory responses. ${ }^{70}$ Passive administration of antigen-specific or non-specific IgA reduced airways responsiveness and lung eosinophilia ${ }^{71}$ or allergic rhinitis. ${ }^{72}$ The impact of IgA and SIgA titers during the first 2 years on the development of allergy was also reported. ${ }^{73}$ Reduction of fecal SIgA when compared with mice actively tolerized with the same protein antigen $^{74}$ argue in favor of the importance of SIgA in controlling allergic reactions, yet the role of IgG-based complexes with $\mathrm{Ag}$ 
cannot be excluded. ${ }^{75}$ Furthermore, pIgR knockout mice, which are unable to produce SIgA and have increased intestinal permeability, display a greater systemic immune response toward commensal bacteria, but not toward food antigens, ${ }^{76}$ a phenomenon possibly linked to increased uptake of food antigens. ${ }^{77}$ In the same knockout model, the capacity of mice to trigger oral tolerance and protect against systemic hypersensitivity by the same tolerizing antigen has also been demonstrated; ${ }^{78}$ this suggests a delicate balance between the development of secretory immunity and mucosal leakiness.

Moreover, first-line mucosal defenses were documented in grass pollen immunotherapy. ${ }^{79} \mathrm{CD} 89$ (Fc $\alpha$ RI) cross-linking by IgA inhibits FceRI-dependent activation of mast cells, and diminishes allergic asthma in transgenic mice expressing the human receptor. ${ }^{80}$ Aggregated milk allergens are taken up by Peyer's patches rather than by classical epithelial cell-mediated phagocytosis: ${ }^{81}$ this resulted in the increased production of both Th2-associated IgE and SIgA. Although the authors con- cluded on enhanced sensitization, they neglected to consider the increase in luminal SIgA, and the contribution of the $\mathrm{Ab}$ in neutralizing the allergens, ultimately limiting allergic responses.

However, the presence of allergen-specific SIgA is not always augmented in successfully tolerized animals, and can even be present in large amounts in sensitized animals without conferring protection. ${ }^{82}$ Moreover, the importance of SIgA against allergic diseases remains unclear with respect to recent clinical studies; patients with IgA deficiency display an increased risk of food hypersensitivity at the age of 4 years solely, ${ }^{52}$ whereas in another cohort, IgA deficiency does not show any correlation with food allergy. ${ }^{83}$ It is an open question whether the production of compensatory SIgM can explain this discrepancy. The sum of these data suggests that SIgA production is more critical for homeostasis toward commensal bacteria than food antigens. Additional studies are required to clarify the importance of SIgA in the maintenance of oral tolerance, and hence the integrity of the intestinal barrier.

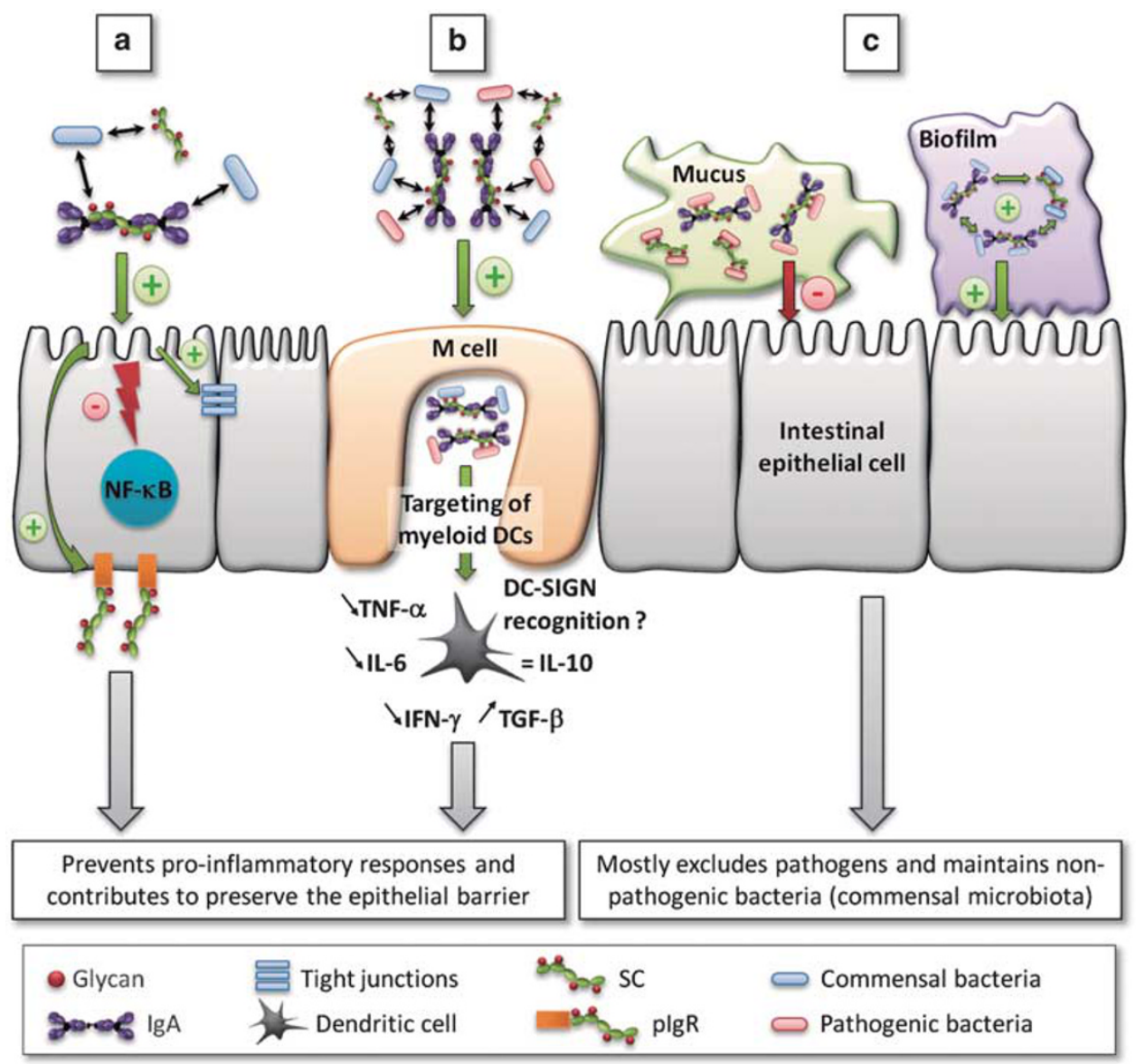

Figure 3 Multi-functional interactions between SIgA and pathogenic and non-pathogenic bacteria in the intestinal mucosa. In all pathways, pathogenic and non-pathogenic bacteria are coated by SIgA (depicted as a dimer with bound SC) in a Fab-specific or in a Fab-independent, glycanmediated manner. (a) Enhanced interaction between SIgA-coated commensal bacteria and the epithelium reinforces its barrier function through multiple mechanisms, including reinforcement of tight junctions, overproduction of plgR, and reduction in nuclear translocation of NF- $\mathrm{kB}$. (b) SIgAbased immune complexes with commensal and/or pathogenic bacteria are taken up by M cells wherein they are targeted to underlying myeloid DCs, possibly upon binding to DC-SIGN, resulting in the downregulation of local proinflammatory responses. (c) SIgA, as well as free SC (not depicted in the figure), may have a role of "selection" by excluding pathogenic bacteria off the epithelial surface through anchoring within mucus and favoring biofilm formation of non-pathogenic bacteria in the space in close contact with epithelial cells. + : activatory effect; - : inhibitory effect. DC, dendritic cell; IFN- $\gamma$,

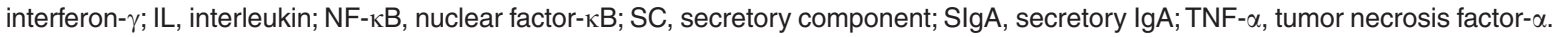




\section{CD: SIgA as a Trojan horse}

An exception to the generally accepted function of immune exclusion of SIgA is the observation that the antibody acts as a Trojan horse in people suffering from CD. In genetically susceptible individuals with CD, complexes of luminal specific SIgA antibodies and gluten-derived deamidated gliadin peptides are retro-transcytosed across epithelial cells, leading to the basal delivery of intact, highly reactive peptides that stimulate inflammatory processes through activation of target $\mathrm{CD} 4{ }^{+} \mathrm{T}$ cells. ${ }^{84}$ This abnormal intestinal transport is mediated by the recognition of SIgA-gliadin complexes by the transferrin receptor (TfR, CD71) expressed at high levels on the apical surface of intestinal epithelial cells in CD patients. The disease thus seems a deficient confining of SIgA-based immune complexes resulting from the misaddressing of CD71, and its subsequent fortuitous capacity to transcytose toxic gliadin peptides. However, it remains unclear why in the physiological context the large excess of luminal SIgA displaying multiple specificities cannot prevent most of receptor-mediated endocytosis, although effective competition occurs in the presence of polymeric IgA, SIgA, or soluble CD71 in Ussing chambers in vitro.

\section{Recognition of SIgA-antigen complexes by antigen- presenting cells}

The SED region is the primary depot for antigens and SIgAantigen complexes after M-cell transcytosis. ${ }^{68,85}$ Although both mouse- and human-derived DCs are capable of binding and internalizing SIgA, the specific IgA receptor(s) on DCs involved in SIgA recognition have not been fully identified. Heystek et al. ${ }^{86}$ speculated that the interaction of SIgA with human monocyte-derived DCs occurs through a member(s) of the C-type lectin family of receptors. We recently tested this hypothesis and found that human colostral SIgA is recognized (and internalized) by human DC-specific intracellular adhesion molecule-3 grabbing non-integrin (DC-SIGN), as well as the mannose receptor (MR; CD206). ${ }^{87} \mathrm{DC}-\mathrm{SIGN}$ (but not the MR) is expressed on myeloid DCs in the SED region, arguing for a possible role for a subset of C-type lectins in immune sampling. ${ }^{88}$ The mouse homolog of DC-SIGN, SIGNR1 (specific intracellular adhesion molecule-3 grabbing non-integrin-related-1; CD209b) has a binding specificity similar to human DC-SIGN, ${ }^{89}$ and is similarly expressed on intestinal CD $11 \mathrm{c}^{+} \mathrm{CD} 11 \mathrm{~b}^{+} \mathrm{DCs} .{ }^{90}$ On the other hand, the possibility that other receptor(s) besides DC-SIGN are involved in sampling SIgA cannot be excluded. In the mouse, e.g., it was noted that the association of SIgA with Peyer's patch DCs was largely unaffected by the glycosylation state of IgA. ${ }^{91}$

\section{CONCLUSIONS}

Besides its well-documented capacity to protect the intestinal epithelium from toxins, viruses, and pathogenic bacteria, SIgA demonstrates an array of other activities that are integral to the maintenance of mucosal homeostasis (Figure 3). SIgA influences the composition of the intestinal microbiota, downregulates proinflammatory responses normally associated with the uptake of highly pathogenic bacteria and potentially allergenic antigens, and promotes the retro-transport of antigens across the intestinal epithelium to DC subsets in GALTs. SIgA's ability to multitask is due in large part to it intrinsic complexity, particularly the diverse glycan arrays on both polymeric IgA and bound SC. Our increasing ability to biochemically dissect SIgA into its individual components and then test them in defined animal models will ultimately permit us to ascribe specific tasks to SIgA in molecular detail.

\section{ACKNOWLEDGMENTS}

The research topics presented in this review are supported by research grants from the Swiss Science Research Foundation (3200-122039) to B.C. and the National Institutes of Health (HD061916) to N.J.M. We are grateful to Dr Steve Forbes and the Wadsworth Center's Electron Microscopy Core facility for Figure 1.

\section{DISCLOSURE}

The authors declared no conflict of interest.

(c) 2011 Society for Mucosal Immunology

\section{REFERENCES}

1. Brandtzaeg, P. Function of mucosa-associated lymphoid tissue in antibody formation. Immunol. Invest. 39, 303-355 (2010).

2. He, B. et al. Intestinal bacteria trigger T cell-independent immunoglobulin A(2) class switching by inducing epithelial-cell secretion of the cytokine APRIL. Immunity 26, 812-826 (2007).

3. Newberry, R.D. \& Lorenz, R.G. Organizing a mucosal defense. Immunol. Rev. 206, 6-21 (2005).

4. Apter, F.M., Lencer, W.I., Finkelstein, R.A., Mekalanos, J.J. \& Neutra, M.R. Monoclonal immunoglobulin A antibodies directed against cholera toxin prevent the toxin-induced chloride secretory response and block toxin binding to intestinal epithelial cells in vitro. Infect. Immunol. 61, 5271-5278 (1993)

5. Helander, A., Miller, C.L., Myers, K.S., Neutra, M.R. \& Nibert, M.L. Protective immunoglobulin $A$ and $G$ antibodies bind to overlapping intersubunit epitopes in the head domain of type 1 reovirus adhesin sigma1. J. Virol. 78, 10695-10705 (2004).

6. Hutchings, A.B. et al. Secretory immunoglobulin A antibodies against the sigma1 outer capsid protein of reovirus type 1 Lang prevent infection of mouse Peyer's patches. J. Virol. 78, 947-957 (2004).

7. Mantis, N.J., McGuinness, C.R., Sonuyi, O., Edwards, G. \& Farrant, S.A. Immunoglobulin $A$ antibodies against ricin $A$ and $B$ subunits protect epithelial cells from ricin intoxication. Infect. Immunol. 74, 3455-3462 (2006).

8. Stubbe, H., Berdoz, J., Kraehenbuhl, J.P. \& Corthésy, B. Polymeric IgA is superior to monomeric $\lg A$ and $\lg G$ carrying the same variable domain in preventing Clostridium difficile toxin A damaging of T84 monolayers. J. Immunol. 164, 1952-1960 (2000).

9. Uren, T.K. et al. Vaccine-induced protection against gastrointestinal bacterial infections in the absence of secretory antibodies. Eur. J. Immunol. 35, 180-188 (2005).

10. Lycke, N., Erlandsson, L., Ekman, L., Schon, K. \& Leanderson, T. Lack of $\mathrm{J}$ chain inhibits the transport of gut IgA and abrogates the development of intestinal antitoxic protection. J. Immunol. 163, 913-919 (1999).

11. Apter, F.M. et al. Analysis of the roles of antilipopolysaccharide and anticholera toxin immunoglobulin $\mathrm{A}(\lg \mathrm{A})$ antibodies in protection against Vibrio cholerae and cholera toxin by use of monoclonal IgA antibodies in vivo. Infect. Immunol. 61, 5279-5285 (1993).

12. Silvey, K.J., Hutchings, A.B., Vajdy, M., Petzke, M.M. \& Neutra, M.R. Role of immunoglobulin A in protection against reovirus entry into murine Peyer's patches. J. Virol. 75, 10870-10879 (2001).

13. Helander, A. et al. The viral sigma1 protein and glycoconjugates containing alpha2-3-linked sialic acid are involved in type 1 reovirus adherence to M cell apical surfaces. J. Virol. 77, 7964-7977 (2003).

14. Deplancke, B. \& Gaskins, H.R. Microbial modulation of innate defense: goblet cells and the intestinal mucus layer. Am. J. Clin. Nutr. 73, 1131S-1141S (2001). 
15. Lievin-Le Moal, V. \& Servin, A.L. The front line of enteric host defense against unwelcome intrusion of harmful microorganisms: mucins, antimicrobial peptides, and microbiota. Clin. Microbiol. Rev. 19, 315-337 (2006).

16. Mantis, N.J. \& Forbes, S.J. Secretory IgA: arresting microbial pathogens at epithelial borders. Immunol. Invest. 39, 383-406 (2010).

17. Stokes, C.R., Soothill, J.F. \& Turner, M.W. Immune exclusion is a function of lgA. Nature 255, 745-746 (1975).

18. Brandtzaeg, P. Mucosal immunity: induction, dissemination, and effector functions. Scand. J. Immunol. 70, 505-515 (2009).

19. Forbes, S.J., Eschmann, M. \& Mantis, N.J. Inhibition of Salmonella enterica serovar typhimurium motility and entry into epithelial cells by a protective antilipopolysaccharide monoclonal immunoglobulin A antibody. Infect. Immunol. 76, 4137-4144 (2008).

20. Michetti, P., Mahan, M.J., Slauch, J.M., Mekalanos, J.J. \& Neutra, M.R. Monoclonal secretory immunoglobulin A protects mice against oral challenge with the invasive pathogen Salmonella typhimurium. Infect. Immunol. 60, 1786-1792 (1992).

21. Boullier, S. et al. Secretory lgA-mediated neutralization of Shigella flexneri prevents intestinal tissue destruction by down-regulating inflammatory circuits. J. Immunol. 183, 5879-5885 (2009).

22. Phalipon, A. et al. Secretory component: a new role in secretory IgA-mediated immune exclusion in vivo. Immunity 17, 107-115 (2002).

23. McGuckin, M.A., Linden, S.K., Sutton, P. \& Florin, T.H. Mucin dynamics and enteric pathogens. Nat. Rev. Microbiol. 9, 265-278 (2011).

24. Kraehenbuhl, J.P. \& Neutra, M.R. Molecular and cellular basis of immune protection of mucosal surfaces. Physiol. Rev. 72, 853-879 (1992).

25. Corthésy, B. et al. Rotavirus anti-VP6 secretory immunoglobulin A contributes to protection via intracellular neutralization but not via immune exclusion. J. Virol. 80, 10692-10699 (2006).

26. lankov, I.D. et al. Protective efficacy of IgA monoclonal antibodies to $\mathrm{O}$ and $\mathrm{H}$ antigens in a mouse model of intranasal challenge with Salmonella enterica serotype Enteritidis. Microbes Infect. 6, 901-910 (2004).

27. Michetti, P. et al. Monoclonal immunoglobulin A prevents adherence and invasion of polarized epithelial cell monolayers by Salmonella typhimurium. Gastroenterology 107, 915-923 (1994).

28. Forbes, S.J., Bumpus, T., McCarthy, E.A., Corthésy, B. \& Mantis, N.J. Transient suppression of Shigella flexneri Type 3 secretion by a protective O-antigen-specific monoclonal lgA. MBio 2, e00042-11 (2011).

29. Dallas, S.D. \& Rolfe, R.D. Binding of Clostridium difficile toxin A to human milk secretory component. J. Med. Microbiol. 47, 879-888 (1998).

30. Mantis, N.J., Farrant, S.A. \& Mehta, S. Oligosaccharide side chains on human secretory IgA serve as receptors for ricin. J. Immunol. 172, 6838-6845 (2004).

31. Mestecky, J. \& Russell, M.W. Specific antibody activity, glycan heterogeneity and polyreactivity contribute to the protective activity of S-IgA at mucosal surfaces. Immunol. Lett. 124, 57-62 (2009).

32. Perrier, C., Sprenger, N. \& Corthésy, B. Glycans on secretory component participate in innate protection against mucosal pathogens. J. Biol. Chem 281, 14280-14287 (2006).

33. Royle, L. et al. Secretory IgA N- and O-glycans provide a link between the innate and adaptive immune systems. J. Biol. Chem. 278, 20140-20153 (2003).

34. Schroten, H. et al. Fab-independent antiadhesion effects of secretory immunoglobulin A on S-fimbriated Escherichia coli are mediated by sialyloligosaccharides. Infect. Immunol. 66, 3971-3973 (1998).

35. Wold, A.E. et al. Secretory immunoglobulin A carries oligosaccharide receptors for Escherichia coli type 1 fimbrial lectin. Infect. Immunol. 58, 3073-3077 (1990)

36. Lu, L., Lamm, M.E., Li, H., Corthésy, B. \& Zhang, J.R. The human polymeric immunoglobulin receptor binds to Streptococcus pneumoniae via domains 3 and 4. J. Biol. Chem. 278, 48178-48187 (2003).

37. Murthy, A.K. et al. Mannose-containing oligosaccharides of non-specific human secretory immunoglobulin A mediate inhibition of Vibrio cholerae biofilm formation. PLoS One 6, e16847 (2011).

38. Mathias, A. \& Corthésy, B. Recognition of Gram-positive intestinal bacteria by Hybridoma- and Colostrum-derived secretory immunoglobulin a is mediated by carbohydrates. J. Biol. Chem. 286, 17239-17247 (2011).

39. Mathias, A. et al. Potentiation of polarized intestinal Caco-2 cell responsiveness to probiotics complexed with secretory lgA. J. Biol. Chem. 285, 33906-33913 (2010).
40. Sekirov, I., Russell, S.L., Antunes, L.C. \& Finlay, B.B. Gut microbiota in health and disease. Physiol. Rev. 90, 859-904 (2010).

41. Walter, J. \& Ley, R.E. The human gut microbiome: ecology and recent evolutionary changes. Annu. Rev. Microbiol. (2011).

42. Cebra, J.J. Influences of microbiota on intestinal immune system development. Am. J. Clin. Nutr. 69, 1046S-1051S (1999).

43. Jiang, H.Q. et al. Interactions of commensal gut microbes with subsets of B- and T-cells in the murine host. Vaccine 22, 805-811 (2004).

44. Stoel, M. et al. Restricted IgA repertoire in both B-1 and B-2 cell-derived gut plasmablasts. J. Immunol. 174, 1046-1054 (2005).

45. Gardby, E. et al. The influence of costimulation and regulatory CD4+ T cells on intestinal IgA immune responses. Dev. Immunol. 6, 53-60 (1998).

46. Macpherson, A.J. et al. A primitive T cell-independent mechanism of intestinal mucosal IgA responses to commensal bacteria. Science $\mathbf{2 8 8}$, 2222-2226 (2000).

47. Macpherson, A.J. \& Uhr, T. Induction of protective IgA by intestinal dendritic cells carrying commensal bacteria. Science 303, 1662-1665 (2004).

48. Hapfelmeier, S. et al. Reversible microbial colonization of germ-free mice reveals the dynamics of IgA immune responses. Science $\mathbf{3 2 8 , 1 7 0 5 - 1 7 0 9}$ (2010).

49. Fagarasan, S. et al. Critical roles of activation-induced cytidine deaminase in the homeostasis of gut flora. Science 298, 1424-1427 (2002).

50. Yel, L. Selective IgA deficiency. J. Clin. Immunol. 30, 10-16 (2010).

51. Meini, A. et al. Prevalence and diagnosis of celiac disease in IgA-deficient children. Ann. Allergy Asthma Immunol. 77, 333-336 (1996).

52. Janzi, M. et al. Selective IgA deficiency in early life: association to infections and allergic diseases during childhood. Clin. Immunol. 133, 78-85 (2009).

53. Brandtzaeg, P. et al. The clinical condition of lgA-deficient patients is related to the proportion of lgD- and lgM-producing cells in their nasal mucosa. Clin. Exp. Immunol. 67, 626-636 (1987).

54. Corthésy, B. Roundtrip ticket for secretory IgA: role in mucosal homeostasis? J. Immunol. 178, 27-32 (2007).

55. van der Waaij, L.A., Limburg, P.C., Mesander, G. \& van der Waaij, D. In vivo IgA coating of anaerobic bacteria in human faeces. Gut 38, 348-354 (1996).

56. Tsuruta, T. et al. The amount of secreted IgA may not determine the secretory IgA coating ratio of gastrointestinal bacteria. FEMS Immunol. Med. Microbiol. 56, 185-189 (2009).

57. Peterson, D.A., McNulty, N.P., Guruge, J.L. \& Gordon, J.I. IgA response to symbiotic bacteria as a mediator of gut homeostasis. Cell Host Microbe 2 , 328-339 (2007).

58. Zitomersky, N.L., Coyne, M.J. \& Comstock, L.E. Longitudinal analysis of the prevalence, maintenance, and lgA response to species of the order bacteroidales in the human gut. Infect. Immunol. 79, 2012-2020 (2011).

59. Friman, V. et al. Decreased expression of mannose-specific adhesins by Escherichia coli in the colonic microflora of immunoglobulin A-deficient individuals. Infect. Immunol. 64, 2794-2798 (1996).

60. Bollinger, R.R. et al. Human secretory immunoglobulin A may contribute to biofilm formation in the gut. Immunology 109, 580-587 (2003).

61. Bollinger, R.R. et al. Secretory IgA and mucin-mediated biofilm formation by environmental strains of Escherichia coli: role of type 1 pili. Mol. Immunol. 43, 378-387 (2006).

62. Costerton, J.W., Lewandowski, Z., Caldwell, D.E., Korber, D.R. \& LappinScott, H.M. Microbial biofilms. Annu. Rev. Microbiol. 49, 711-745 (1995).

63. Palestrant, D. et al. Microbial biofilms in the gut: visualization by electron microscopy and by acridine orange staining. Ultrastruct. Pathol. 28, 23-27 (2004).

64. Lebeer, S., Claes, I.J., Verhoeven, T.L., Vanderleyden, J. \& De Keersmaecker, S.C. Exopolysaccharides of Lactobacillus rhamnosus GG form a protective shield against innate immune factors in the intestine. Microb. Biotechnol. 4, 368-374 (2011).

65. Mantis, N.J. et al. Selective adherence of IgA to murine Peyer's patch M cells: evidence for a novel IgA receptor. J. Immunol. 169, 1844-1851 (2002).

66. Weltzin, R. et al. Binding and transepithelial transport of immunoglobulins by intestinal M cells: demonstration using monoclonal IgA antibodies against enteric viral proteins. J. Cell Biol. 108, 1673-1685 (1989).

67. Duc, M., Johansen, F.E. \& Corthésy, B. Antigen binding to secretory immunoglobulin A results in decreased sensitivity to intestinal proteases and increased binding to cellular Fc receptors. J. Biol. Chem. 285, 953-960 (2010). 
68. Favre, L., Spertini, F. \& Corthésy, B. Secretory lgA possesses intrinsic modulatory properties stimulating mucosal and systemic immune responses. J. Immunol. 175, 2793-2800 (2005).

69. Sansonetti, P.J., Arondel, J., Cantey, J.R., Prevost, M.C. \& Huerre, M. Infection of rabbit Peyer's patches by Shigella flexneri: effect of adhesive or invasive bacterial phenotypes on follicle-associated epithelium. Infect. Immunol. 64, 2752-2764 (1996).

70. Smits, H.H. et al. Cholera toxin B suppresses allergic inflammation through induction of secretory IgA. Mucosal Immunol. 2, 331-339 (2009).

71. Schwarze, J. et al. Antigen-specific immunoglobulin-A prevents increased airway responsiveness and lung eosinophilia after airway challenge in sensitized mice. Am. J. Respir. Crit. Care Med. 158, 519-525 (1998).

72. Heikkinen, T. et al. Intranasally administered immunoglobulin for the prevention of rhinitis in children. Pediatr. Infect. Dis. J. 17, 367-372 (1998).

73. Bottcher, M.F., Haggstrom, P., Bjorksten, B. \& Jenmalm, M.C. Total and allergen-specific immunoglobulin A levels in saliva in relation to the development of allergy in infants up to 2 years of age. Clin. Exp. Allergy 32, 1293-1298 (2002).

74. Frossard, C.P., Hauser, C. \& Eigenmann, P.A. Antigen-specific secretory IgA antibodies in the gut are decreased in a mouse model of food allergy. J. Allergy Clin. Immunol. 114, 377-382 (2004).

75. Mosconi, E. et al. Breast milk immune complexes are potent inducers of oral tolerance in neonates and prevent asthma development. Mucosal Immunol. 3, 461-474 (2010).

76. Johansen, F.E. et al. Absence of epithelial immunoglobulin A transport, with increased mucosal leakiness, in polymeric immunoglobulin receptor/ secretory component-deficient mice. J. Exp. Med. 190, 915-922 (1999).

77. Sait, L.C. et al. Secretory antibodies reduce systemic antibody responses against the gastrointestinal commensal flora. Int. Immunol. 19, 257-265 (2007).

78. Karlsson, M.R., Johansen, F.E., Kahu, H., Macpherson, A. \& Brandtzaeg, P. Hypersensitivity and oral tolerance in the absence of a secretory immune system. Allergy 65, 561-570 (2010).

79. Pilette, C. et al. Grass pollen immunotherapy induces an allergen-specific IgA2 antibody response associated with mucosal TGF-beta expression. J. Immunol. 178, 4658-4666 (2007).
80. Pasquier, B. et al. Identification of FcalphaRI as an inhibitory receptor that controls inflammation: dual role of FcRgamma ITAM. Immunity 22, 31-42 (2005)

81. Roth-Walter, F. et al. Pasteurization of milk proteins promotes allergic sensitization by enhancing uptake through Peyer's patches. Allergy 63, 882-890 (2008).

82. Perrier, C., Thierry, A.C., Mercenier, A. \& Corthésy, B. Allergen-specific antibody and cytokine responses, mast cell reactivity and intestinal permeability upon oral challenge of sensitized and tolerized mice. Clin. Exp. Allergy 40, 153-162 (2010).

83. Aghamohammadi, A. et al. IgA deficiency: correlation between clinical and immunological phenotypes. J. Clin. Immunol. 29, 130-136 (2009).

84. Matysiak-Budnik, T. et al. Secretory IgA mediates retrotranscytosis of intact gliadin peptides via the transferrin receptor in celiac disease. J. Exp. Med. 205, 143-154 (2008).

85. Rey, J., Garin, N., Spertini, F. \& Corthésy, B. Targeting of secretory IgA to Peyer's patch dendritic and T cells after transport by intestinal $\mathrm{M}$ cells. J Immunol. 172, 3026-3033 (2004).

86. Heystek, H.C., Moulon, C., Woltman, A.M., Garonne, P. \& van Kooten, C. Human immature dendritic cells efficiently bind and take up secretory IgA without the induction of maturation. J. Immunol. 168, 102-107 (2002).

87. Baumann, J., Park, C.G. \& Mantis, N.J. Recognition of secretory lgA by DC-SIGN: implications for immune surveillance in the intestine. Immunol. Lett. 131, 59-66 (2010).

88. Jameson, B. et al. Expression of DC-SIGN by dendritic cells of intestinal and genital mucosae in humans and rhesus macaques. J. Virol. 76, 1866-1875 (2002).

89. Galustian, C. et al. High and low affinity carbohydrate ligands revealed for murine SIGN-R1 by carbohydrate array and cell binding approaches, and differing specificities for SIGN-R3 and langerin. Int. Immunol. 16, 853-866 (2004).

90. Zhou, Y. et al. Oral tolerance to food-induced systemic anaphylaxis mediated by the C-type lectin SIGNR1. Nat. Med. 16, 1128-1133 (2010).

91. Kadaoui, K.A. \& Corthésy, B. Secretory IgA mediates bacterial translocation to dendritic cells in mouse Peyer's patches with restriction to mucosal compartment. J. Immunol. 179, 7751-7757 (2007). 